\title{
Efecto del pH en emulsiones o/w formuladas con proteína de patata y quitosano
}

\author{
Por N. Calero*, J. Muñoz y A. Guerrero \\ Departamento de Ingeniería Química. Facultad de Química. Universidad de Sevilla. \\ c/ P. García González, 1, E41012-Sevilla (España). \\ *Correspondencia: nuriacalero@us.es
}

\section{RESUMEN}

\author{
Efecto del $\mathrm{pH}$ en emulsiones o/w formuladas con \\ proteína de patata y quitosano
}

Las emulsiones con ingredientes saludables están adquiriendo más popularidad en el mundo desarrollado, ya que el consumidor es consciente de la relación existente entre algunas enfermedades y las dietas ricas en grasas. En este trabajo se estudian emulsiones $\mathrm{O} / \mathrm{W}$ formuladas con aceite de girasol alto oleico, una proteína vegetal (proteína de patata) y quitosano. Concretamente, se estudia la influencia del pH en la preparación de emulsiones que contenían $40 \%$ de aceite de girasol alto oleico.

Se prepararon suspensiones de la proteína en un intervalo de valores de $\mathrm{pH}$ comprendido entre 2 y 11,5 , observándose un aumento de solubilidad de la proteína al aumentar el $\mathrm{pH}$.

El aumento del pH de la emulsión provocó un aumento de la viscoelasticidad, que quedaba reflejado en un incremento de los valores de los módulos de almacenamiento y pérdida, respectivamente, así como, en un cambio progresivo en la forma del espectro mecánico. Por otro lado, las curvas de flujo mostraron propiedades pseudoplásticas y un aumento de los valores de la viscosidad con el $\mathrm{pH}$.

Las medidas de potencial $Z$ revelaron la existencia de interacciones atractivas entre la proteína de patata y el quitosano que aumentaban progresivamente con el pH.

Los resultados obtenidos pueden explicar la existencia de un complejo proteína de patata-quitosano que se coloca alrededor de las gotas de aceite y que justifica el aumento de estabilidad física.

PALABRAS CLAVE: Aceite de girasol alto oleico Emulsión - Proteína de patata - Quitosano - Reología.

\section{SUMMARY}

Effect of $\mathrm{pH}$ on o/w emulsions formulated with potato protein and chitosan

Emulsions formulated with healthy ingredients are becoming increasingly popular in the developed world, due to the consumer concern about diseases related to fat and to fatty diets. This study focuses on the $\mathrm{O} / \mathrm{W}$ emulsions formulated with high-oleic sunflower oil, vegetable protein (potato protein) and chitosan; and deals with the role of $\mathrm{pH}$ in the preparation of $40 \mathrm{wt} \%$ high-oleic sunflower oil in water emulsions. First of all, potato protein suspensions were prepared in a pH range from 2.0 to 11.5 and enhanced protein solubility was observed with increasing $\mathrm{pH}$.

An increase in $\mathrm{pH}$ emulsion resulted in more viscoelasticity as demonstrated by the greater values for the storage and lost moduli derived from small amplitude oscillatory shear measurements as well as by the progressive change in the shape of the mechanical spectrum. Flow curves showed shear thinning properties and increasing viscosity values with $\mathrm{pH}$.

Z-potential measurements revealed that attractive interactions between potato protein and chitosan progressively increased with $\mathrm{pH}$.

The results obtained are consistent with the occurrence of a protein-polysaccharide complex around the interface of oil droplets, which yielded enhanced physical stability.

KEY-WORDS: Chitosan - Emulsion - High oleic sunflower oil - Potato protein -Rheology.

\section{INTRODUCCIÓN}

Las emulsiones aceite-en-agua consisten en una fase oleosa no polar que se encuentra formando gotas dispersas en una fase acuosa polar. En general, las emulsiones son sistemas termodinámicamente inestables y las fases que la componen tienden a separarse por diferentes mecanismos (McClements, 2005). Sin embargo, las emulsiones pueden estabilizarse cinéticamente, incluso por largos periodos de tiempo, usando dos tipos de sustancias, los emulsionante y los estabilizantes que retardan o inhiben los mecanismos de desestabilización.

Los emulsionantes son moléculas que poseen actividad superficial y que por ello, tienden a adsorberse en la superficie de las gotas formando una barrera protectora que previene la agregación entre ellas. Los emulsionantes que se usan generalmente son proteínas anfifílicas, polisacáridos, tensioactivos de bajo peso molecular y fosfolípidos. Por otro lado, los estabilizantes se usan frecuentemente para retardar el proceso de desestabilización de las emulsiones y controlar su textura (McClements y Weiss, 2005). Los polisacáridos que se usan como estabilizantes provocan un aumento en la viscosidad de la emulsión y además, promueven la formación de una red de gotas de aceite (Dickinson, 2009). Además, algunos polisacáridos poseen actividad superficial y por tanto actúan como excelentes emulsificantes (Dickinson, 2003).

La utilización de mezclas de proteínas y polisacáridos en emulsiones alimentarias puede promover una mejora de las propiedades funcionales de- 
bido a la formación de complejos durante los procesos de pre-emulsificación y/o post-emulsificación (Dickinson, 2003; Schmitt et al., 1998). La formación de los complejos proteína-polisacárido puede ser durante la pre-emulsificación bien por tratamiento de dos hidrocoloides que formen un conjugado covalente o por atracción electrostática entre dos hidrocoloides con carga opuesta que formen complejos coacervados (Dickinson, 2003; Schmitt et al., 1998). Posteriormente, se usa la fase acuosa que contiene el complejo coacervado como emulsificante para formar la emulsión O/W por homogenización con la fase oleosa. Una estrategia alternativa es la formación de complejos proteína-polisacárido durante la post-emulsificación. En este caso, la proteína se absorbe en las interfase de las gotas durante la primera etapa de homogenización y en la etapa posterior, se añade el hidrocoloide de carga opuesta que además proporciona estabilidad por impedimento estérico y finalmente se obtiene una emulsión con gotas rodeadas por bicapas proteína-polisacárido (McClemensts, 2004)

Las interacciones atractivas y repulsivas entre proteínas y polisacáridos dependerán de factores tales como el $\mathrm{pH}$ y la fuerza iónica $(\mu)$ del sistema. Por debajo del pl de las proteínas y con polisacáridos catiónicos, tales como el quitosano, en general ocurrirán interacciones repulsivas, mientras que por encima del pl ocurrirán interacciones atractivas formando complejos coacervados (Dickinson, 2003).

Tradicionalmente, se ha empleado la yema de huevo como emulsionante en las emulsiones alimentarias. Sin embargo, actualmente existe una tendencia creciente al consumo de productos bajos en calorías y más saludables. Por ello en los últimos años se ha estudiado el empleo de emulsionantes alternativos de origen vegetal que puedan sustituir a los de origen animal.

Además, algunas de estas proteínas pueden extraerse a partir de residuos o subproductos de la industria alimentaria como por ejemplo de la producción del almidón (Romero et al., 2011). La proteína de patata presenta una alta calidad nutricional casi similar a la que presenta la proteína del huevo (Bartova y Barta, 2009; Van Gelder y Vonk, 1980). Sin embargo, a pesar de las ventajas derivadas del uso de la proteína de patata en emulsiones alimentarias, existen algunos inconvenientes derivados de la desnaturalización que sufre ésta durante el proceso industrial (Knorr, 1980) lo que le confiere baja solubilidad y capacidad limitada para estabilizar emulsiones (Van Koningsveld et al., 2006). Este hecho haría necesario el uso de un polisacáridos como estabilizante que refuerce la capacidad emulsionante de la proteína de patata cuando se usa en emulsiones alimentarias.

El quitosano es un amino-polisacárido natural que presenta numerosas propiedades fisiológicas (Borderías et al., 2005; Muzzarelli, 2009). Por ello, el uso del quitosano en emulsiones no es solo interesante por su papel como estabilizante sino que también le aporta un valor añadido a la emulsión. De hecho, el uso del quitosano como estabilizante en emulsiones alimentarias ha despertado un significativo interés en la ciencia de alimentos (Ogawa et al., 2003a; Ogawa et al., 2003b; Ogawa et al., 2004; Klinkesorn y McClements, 2009; Chuah et al., 2009; Laplante, et al., 2005; Speiciene, et al., 2007). La acción estabilizante del quitosano en emulsiones tiene lugar no sólo porque provoca un aumento de viscosidad en la fase continua o porque promueve la formación de una red de gotas de aceite, sino que también posee actividad superficial que refuerza la actividad emulsificante cuando se emplea en combinación con una proteína (Calero et al., 2010).

Por otro lado, el aceite de girasol alto oleico es conocido por su alto contenido en ácidos monoinsaturados. Se ha demostrado que existe una relación directa entre las dietas ricas en grasas saturadas y el riesgo de sufrir enfermedades del corazón. Por ello, se recomienda disminuir en la dieta el consumo de grasas saturadas y reemplazarlas por carbohidratos o grasas insaturadas (Krauss, 2000).

Este trabajo es parte de un estudio más amplio enfocado a investigar el uso de ingredientes saludables alternativos a los tradicionales tales como a) aceite de girasol alto oleico del que caben destacar sus beneficios nutricionales, b) proteína de patata obtenida como subproducto del proceso de obtención industrial del almidón, lo que aporta un valor añadido a su uso como estabilizante en emulsiones, y c) quitosano que además de aportar beneficios nutricionales debido a sus propiedades fisiológicas, puede favorecer la formación de una doble capa alrededor de las gotas, teniendo en cuenta su naturaleza catiónica por debajo del punto isoeléctrico de la proteína de patata. En un trabajo publicado anteriormente se estudió la influencia de la concentración de quitosano sobre las propiedades reológicas, estructurales y la estabilidad de las emulsiones. Por un lado, las medidas reológicas demostraron ser una herramienta útil para prever tanto fenómenos de desestabilización como para detectar el mecanismo por el que se desestabiliza. Por otro lado, se pudo comprobar que el quitosano se colocaba preferentemente sobre las capas de proteína adsorbidas en la interfase de las gotas a través de técnicas de microscopía óptica confocal y electrónica de barrido. Este hecho estaba relacionado con la formación del complejo proteína-polisacárido por interacciones electrostáticas (Calero et al., 2013).

El objetivo específico del trabajo actual ha sido el estudio del efecto del $\mathrm{pH}$ en las propiedades reológicas, microestructura y potencial zeta de las emulsiones finales.

\section{MATERIALES Y MÉTODO}

\subsection{Materiales}

El aislado de proteína de patata (PP) fue suministrado por Prostatar (Reus, Barcelona, Spain). La composición química del aislado de proteína de pata- 
ta es la siguiente, $80 \pm 2$ wt\% proteína; 3,1 $\pm 0,4 \mathrm{wt} \%$ lípidos; 5,9 \pm 0,6 wt\% carbohidratos; 0,8 $\pm 0,1$ wt $\%$ ceniza; $10 \pm 2 \mathrm{wt} \%$ humedad. El punto isoeléctrico de PP es 4 (Romero, et al., 2011).

El quitosano $(\mathrm{CH})$ de medio peso molecular (MW 190,000-310,000 Da, 75-85\% deacetilado), el acetato sódico anhidro y el ácido acético glacial fueron suministrados por Sigma Aldrich (St. Louis, USA).

\subsection{Preparación de emulsiones aceite-en-agua (O/W)}

Primer Paso. La dispersión de proteína de patata se preparó dispersando 5,0 wt\% de proteína de patata en polvo en agua Milli-Q water. Esta dispersión se agitó durante 10 minutos y posteriormente se le ajustó el $\mathrm{pH}$ a 2, 3, 6, 8, 10 y 11.5 con $1 \mathrm{M}$ $\mathrm{NaOH}$ o $1 \mathrm{M} \mathrm{HCl}$. A continuación, se preparó una emulsión concentrada mezclando durante 3 minutos y $6000 \mathrm{rpm}, 50 \mathrm{wt} \%$ de aceite y $50 \mathrm{wt} \%$ de suspensión de proteína utilizando un emulsificador tipo rotor-estator, IKA T-50 (pre-emulsión 1).

Segundo Paso. El segundo paso consistió en hacer circular 3 veces la pre-emulsión 1 a través de un homogenizador de alta presión (Emulsi-Flex C5, Avestin) a la presión de 1000 bares (preemulsión 2).

Tercer Paso. Las emulsiones finales se obtuvieron mezclando las pre-emulsiones 2 con una disolución de quitosano (2,5 wt\% quitosano, 0,2 M ácido acético y 0,05 $\mathrm{M}$ acetato sódico, $\mathrm{pH}=3,9$ ), usando el emulsificador IKA T-50, a 6000 rpm durante 3 minutos. De esta forma, las emulsiones finales contenían $40 \mathrm{wt} \%$ de aceite de girasol alto oleico, 40 wt\% de dispersión acuosa de proteína de patata ( 2 wt \% proteína de patata) a diferentes $\mathrm{pH}$ y 20 wt\% de disolución de quitosano (0,5 wt\% de quitosano en la emulsión final) (Figura 1)

La adición del quitosano disuelto en el buffer permitía ajustar el $\mathrm{pH}$ de las emulsiones finales dentro de un intervalo típico en productos alimentarios. Conviene puntualizar que a pesar del amplio

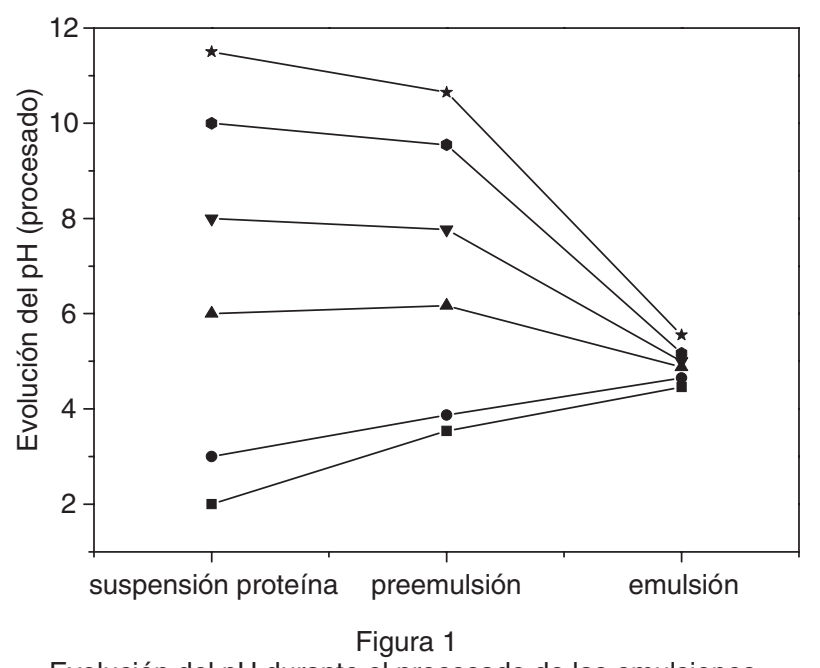

Evolución del pH durante el procesado de las emulsiones. intervalo de valores de $\mathrm{pH}$ de partida, el procedimiento seguido conduce a emulsiones finales con valores de $\mathrm{pH}$ cercanos al punto isoeléctrico (entre $4,5$ y 5,6$)$. Sin embargo, también hay que destacar que no es posible preparar emulsiones estables partiendo de valores de $\mathrm{pH}$ cercanos al punto isoeléctrico.

\subsection{Caracterización reológica}

La caracterización reológica consistió en barridos de esfuerzo y de frecuencia en cizalla oscilatoria y curvas de viscosidad en estado estacionario. Todos los ensayos reológicos se llevaron a cabo en un reómetro de esfuerzo controlado Haake MARS (Thermo-Scientific) con un sistema sensor del tipo placa-placa rugoso (60 mm de diámetro).

Todas las muestras tuvieron la misma historia termomecánica, ya que se mantuvieron en un baño a $25^{\circ} \mathrm{C}$ durante al menos 30 minutos antes del ensayo reológico y permanecieron en la posición de medida durante 10 minutos.

Todos los ensayos reológicos se repitieron por duplicado para cada muestra al día siguiente de su preparación. De ahí que los resultados que se muestran sean el resultado de la media de ambos replicados. La desviación máxima de la media fue siempre menor del $10 \%$ en el caso de G' y G" y del $5 \%$ para las curvas de flujo.

\subsection{Medidas de potencial zeta}

El potencial $z$ de las gotas de aceite de las emulsiones se determinó usando un Nano Zetasizer (Malvern Instruments). Todas las emulsiones se diluyeron con la misma cantidad de agua antes de las medidas para evitar efectos de múltiple Light scattering.

\subsection{Caracterización microestructural}

La microestructura de las emulsiones se observó con un microscopio óptico confocal (CLSM) (Leica TCS SPE, Leica Micro- systems CMS, Mannheim, Alemania).

Para observar las emulsiones en el microscopio óptico confocal, se colocaron muestras de las emulsiones en tubos y a continuación se les añadió Nile Blue para teñir la fase acuosa (quitosano y proteína) y Nile Red en disolución con etanol $(0,01 \%)$ para teñir la fase oleosa. Las muestras se observaron con una lente $63 \mathrm{X}$ con aceite de inmersión y se excitaron con longitudes de ondas de 488 $\mathrm{nm}$ para el Nile Red y $635 \mathrm{~nm}$ para el Nile Blue. La emisión se observó a $10 \mathrm{~nm}$ por encima de la longitud de onda de emisión.

\section{RESULTADOS Y DISCUSIÓN}

La influencia del pH de la suspensión de proteína de patata en el $\xi$-potencial de las gotas de 
las pre-emulsiones 2 y las emulsiones finales se muestra en la Figura 2. El pH juega un papel importante en la ionización de los grupos funcionales de los biopolímeros y por tanto determina la formación del complejo quitosano-proteína de patata. Las proteínas tienen carga positiva neta por debajo de su punto isoeléctrico y por el contrario, tienen carga negativa neta por encima de su punto isoeléctrico.

En este caso, la carga de las gotas de las preemulsiones 2 está sólo condicionada por la carga de la proteína de patata a un pH dado. Por ello, la carga superficial de las partículas de la suspensión de proteína fue positiva a pH 2 y 3 (Figura 1). Por el contrario, la carga superficial de las partículas fue negativa desde $\mathrm{pH} 6$ a pH 11,5. Las medidas de $\xi$-potencial de las emulsiones finales confirman que a esta relación de proteína/quitosano estudiada, había quitosano suficiente para contrarrestar la carga negativa de la proteína. Por ello, a medida que aumenta el $\mathrm{pH}$ de la suspensión de proteína desde 2 a 11,5, el valor del $\xi$-potencial se va haciendo progresivamente menos positivo. Dado que el quitosano y la proteína de patata exhiben cargas opuestas por encima del punto isoeléctrico de la proteína (4), cabe esperar que las macromoléculas de quitosano cargadas con carga positiva formen un complejo con las moléculas de proteína de patata cuya superficie está cargada negativamente. Tal como se ha podido comprobar anteriormente (Calero et al., 2013), la capa protectora proteína-polisacárido que se forma alrededor de las gotas es la responsable del aumento de la estabilidad de la emulsión con el $\mathrm{pH}$. Es interesante destacar que la emulsión con el valor más bajo de $\xi$-potencial es aquella con mayor estabilidad. Esto en principio parece contradictorio si no se tienen en cuenta dos factores fundamentales. En primer lugar, el valor más bajo de $\xi$-potencial se corresponde con el valor más alto de $\mathrm{pH}$ y en estas condiciones,

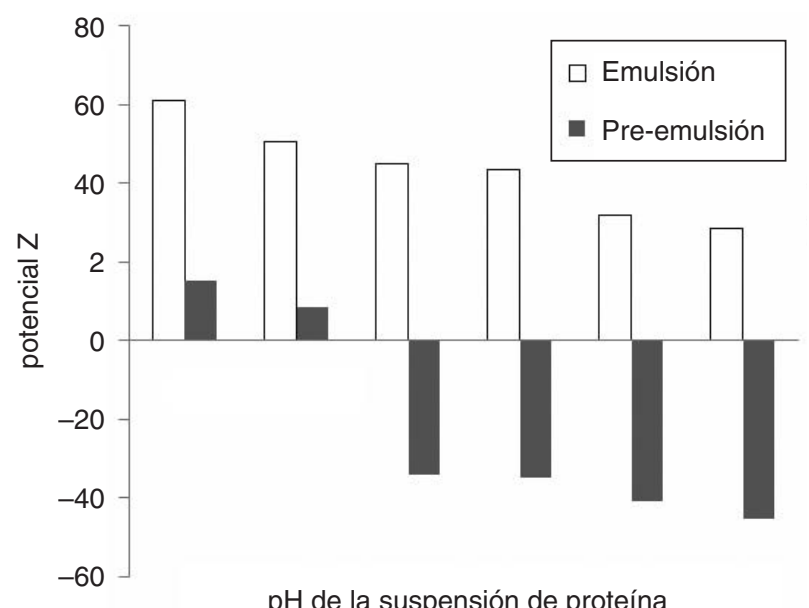

Figura 2

Efecto del $\mathrm{pH}$ de la suspensión de la proteína de patata en e $\zeta$-potencial de la pre-emulsión (proteína de patata, aceite de girasol alto oleico) y de la emulsión final (proteína de patata, aceite de girasol alto oleico y quitosano). existe mayor facilidad de interacción electrostática entre el quitosano cargado positivamente y la proteína cargada negativamente, lo que justifica la reducción del $\xi$-potencial. Este hecho supone una mejora en las interacciones estéricas entre gotas por la formación del complejo proteína-polisacárido que da lugar a una estructura tipo red. Por otro lado, el aumento de pH mejora la solubilidad de la proteína, lo que facilita la adsorción en la interfase y que a su vez favorece la formación del complejo. La existencia de más cantidad de biopolímero en la interfase debe repercutir en sus propiedades mecánicas confiriéndole mayor resistencia frente a fuerzas externas.

Por otro lado, resulta evidente que si bien la formación de la emulsión debe estar condicionada en gran medida por el valor del $\mathrm{pH}$ inicial, es su valor final el que parece ejercer un mayor control sobre la respuesta viscoelástica de la emulsión.

Se realizaron barridos de esfuerzo en cizalla oscilatoria a $1 \mathrm{~Hz}$ para estimar los valores de la amplitud máxima de la función sinusoidal del esfuerzo de cizalla que garantiza un comportamiento viscoelástico lineal.

En la Figura 3 se presentan los valores del módulo elástico (G') y el módulo viscoso (G') en función de la frecuencia angular de todas las emulsiones finales a diferentes valores de $\mathrm{pH}$, que fueron preparadas a partir de las suspensiones de proteína cuyo pH variaba entre 2 y 11,5. En general, las funciones viscoelásticas dinámicas, G' y G", aumentan con el pH de la emulsión en todo el intervalo de frecuencia. De hecho, el aumento de $\mathrm{pH}$ provoca un aumento de la respuesta elástica de las emulsiones. Además, a los pH más bajos, la emulsión exhibe un comportamiento viscoelástico tipo fluido ya que los valores del módulo de pérdidas, G" son inicialmente superiores a los del módulo elástico, G', dando lugar a un punto de cruce entre ambos módulos al aumentar la frecuencia. A medida que aumenta el $\mathrm{pH}$ de la emulsión final, se observa un desplazamiento del punto de cruce hacia frecuencias

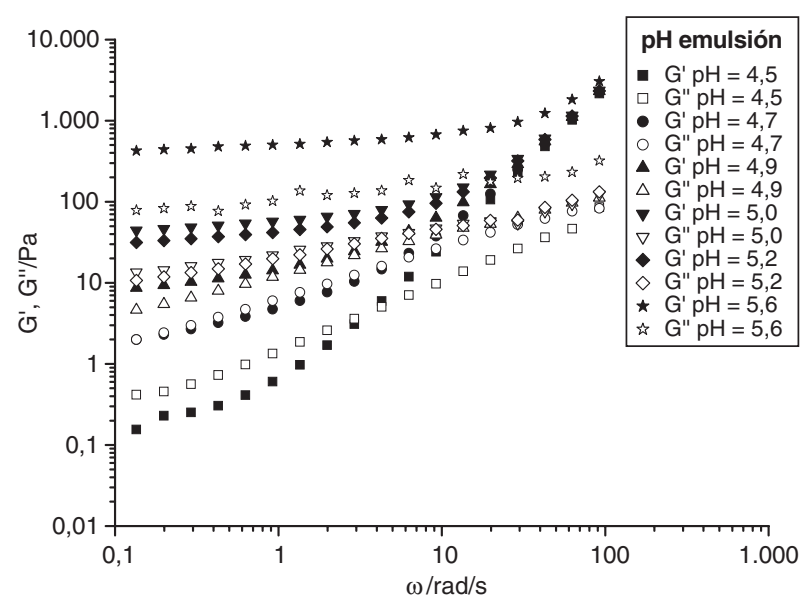

Figura 3

Espectro mecánico en función del pH de la emulsión. Amplitud del esfuerzo: $0.1 \mathrm{~Pa}$. $\mathrm{T}^{\mathrm{a}}=25^{\circ} \mathrm{C}$. 
más altas, seguido de una transición de un comportamiento tipo fluido a un comportamiento tipo gel débil. Este efecto coincide con una disminución progresiva de la dependencia de G' con la frecuencia conforme aumenta el $\mathrm{pH}$, indicando mecanismos de relajación progresivamente más lentos que apuntan a la existencia de una microestructura más desarrollada.

Por otro lado, resulta evidente que si bien la formación de la emulsión debe estar condicionada en gran medida por el valor del $\mathrm{pH}$ inicial, es su valor final el que parece ejercer un mayor control sobre la respuesta viscoelástica de la emulsión.

Resulta interesante destacar el hecho de que el comportamiento tipo gel encontrado a los valores de $\mathrm{pH}$ más elevados difiere en gran medida del encontrado anteriormente para los sistemas quitosano-agua (Calero et al., 2010). Así, incluso a concentraciones muy superiores de quitosano los espectros mecánicos de los sistemas acuosos presentan un comportamiento tipo fluido con valores de módulos muy inferiores (de varios órdenes de magnitud). Este hecho descartaría que la formación del entramado correspondiente al gel elástico se deba exclusivamente a la contribución del quitosano en la fase continua.

Por lo tanto, la respuesta viscoelástica encontrada a $\mathrm{pH}$ elevado puede ser atribuida a la formación de redes de las gotas de fase dispersa cuya superficie se encuentra rodeada de capas de proteína cargadas negativamente y que por tanto interaccionan con las moléculas de quitosano con carga positiva localizadas preferentemente en la fase continua (Calero et al., 2013). La reducción del pH provoca una disminución de las cargas negativas superficiales de la proteína, al aproximarse a su punto isoeléctrico, que produce un claro debilitamiento de estas redes estructurales.

En la Figura 4 se muestran las curvas de flujo de las emulsiones a diferentes $\mathrm{pH}$. Un incremento del $\mathrm{pH}$ en las emulsiones provoca un claro aumento de viscosidad en todo el intervalo de velocidad de cizalla ensayado, lo que concuerda con los re-

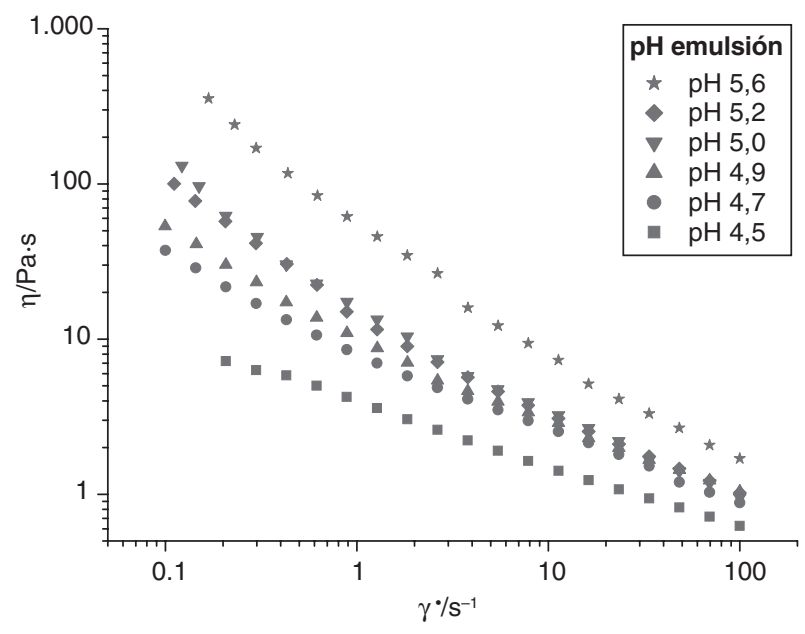

Figure 4

Curvas de flujo en estado estacionario en función del pH de la emulsión a $25^{\circ} \mathrm{C}$.

sultados obtenidos en los ensayos de viscoelasticidad lineal realizados en este estudio.

Por otro lado, todas las emulsiones presentan un comportamiento pseudoplástico en todo el intervalo de velocidad de cizalla estudiado. Además, el aumento de $\mathrm{pH}$ también produce un mayor efecto de destrucción estructural inducido por cizalla, que coincide con el aumento del carácter tipo gel observado.

En la Figura 5 se muestran las micrografías obtenidas por microscopía confocal de la emulsión con mayor $\mathrm{pH}(5,55)$ que es aquella que presentaba mayor estabilidad. En la micrografía de la izquierda se muestra el canal de emisión sólo para el Nile red que colorea la fase oleosa, es decir, el aceite de girasol alto oleico. En la micrografía central se muestra el canal de emisión sólo para el Nile blue que colorea los componentes de la fase acuosa, proteína y quitosano. En este caso, es imposible poder distinguir entre ambos componentes. Por último, en la micrografía de la derecha se muestra la superposición de las dos imágenes anteriores y se ha obtenido mediante el software

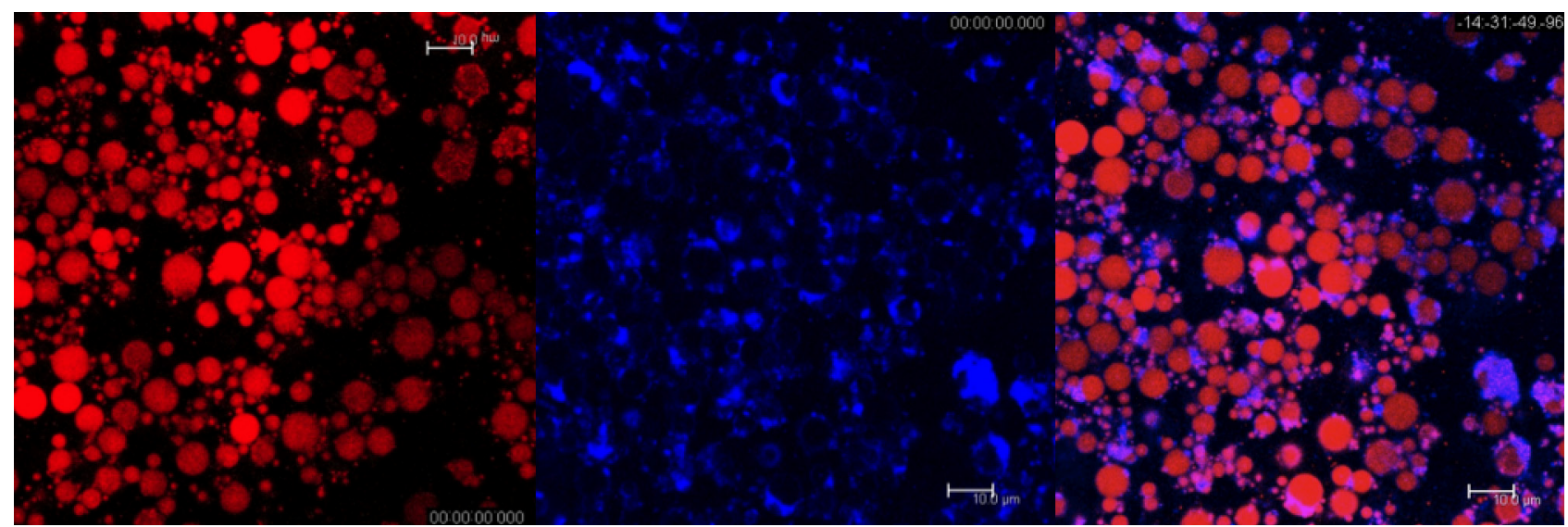

Figura 5

Micrografías obtenidas por microscopía confocal de la emulsión final a $\mathrm{pH} 5.55$ y $25^{\circ} \mathrm{C}$. El color rojo representa al Nile red que tiñe la fase oleosa y el color azul representa al Nile blue que tiñe a la proteína y quitosano. 
del equipo. La micrografía de la derecha donde se distinguen claramente las gotas de aceite, revela la existencia de una emulsión polidispersa. La mayoría de las gotas presentaban un tamaño en un intervalo de 2 a $10 \mu \mathrm{m}$, aunque también se observan gotas más pequeñas. El papel que desempeña el emulsionante puede observarse claramente en la micrografía del centro, donde se distingue la proteína alrededor de las gotas de aceite. Además, esta misma micrografía sugiere que el exceso de proteína formaba agregados en la fase continua. Este hecho puede atribuirse a la baja solubilidad de la proteína. En cualquier caso, el tamaño de los agregados parece ser algo menor que el de las gotas.

\section{CONCLUSIONES}

Las emulsiones formuladas con aceite de girasol alto oleico y proteína de patata y estabilizada con quitosano muestran propiedades viscoelásticas y pasan de un comportamiento viscoelástico tipo fluido a un comportamiento viscoelástico tipo gel a medida que aumenta el pH de la emulsión. El incremento de la respuesta elástica de la emulsión por encima de $\mathrm{pH} 4$ se ha relacionado con la presencia de una microestructura que se desarrolla progresivamente con el aumento de $\mathrm{pH}$. Esto debe estar relacionado con la formación de una capa protectora de complejo proteína de patataquitosano que se colocaba alrededor de las gotas de aceite.

La formación progresiva de esta microestructura resultó también ser eficiente para soportar el efecto del flujo en cizalla estacionaria. La viscosidad aumenta con el pH de la emulsión en todo el intervalo de cizalla estudiado.

Las medidas de potencial $Z$ contribuyen a justificar la estabilidad de la emulsión que se pudo observar visualmente.

\section{AGRADECIMIENTOS}

Los autores desean hacer constar su agradecimiento a la Consejería de Innovación, Ciencia y Tecnología, Junta de Andalucía (proyecto P08AGR-03974) por la financiación aportada.

\section{REFERENCIAS}

Bartova V, Barta J. 2009. Chemical composition and nutritional value of proteína concentrates isolated from potato (Solanum tuberosum L.) fruit juice by precipitation with ethanol or ferric chloride. J. Agr. Food Chem. 57, 9028-9034.

Borderías AJ, Sánchez-Alonso I, Pérez-Mateos M. 2005. New applications of fibres in foods: addition to fishery products. Trends Food Sci. Tech. 16, 458-465

Calero N, Muñoz J, Ramírez P, Guerrero A. 2010. Flow behaviour, linear viscoelasticity and surface proper- ties of chitosan aqueous solutions. Food Hydrocolloids 24, 659-666.

Calero N, Muñoz J, Cox PW. Heuer A, Guerrero A. 2013. Influence of chitosan concentration on the stability, microstructure and rheological properties of $\mathrm{O} / \mathrm{W}$ emulsions formulated with high-oleic sunflower oil and potato protein. Food Hydrocolloids 30, 152162.

Dickinson E. 2009. Hydrocolloids as emulsifiers and emulsion stabilizers. Food hydrocolloids 23, 14731482.

Dickinson E. 2003. Hydrocolloids at interfaces and the influence on the properties of dispersed systems. Food Hydrocolloids 17, 25-39.

Chuah AM, Kuroiwa T, Kobayashi I, Nakajima M. 2009. Effect of chitosan on the stability and properties of modified lecithin stabilized oil-in-water monodisperse emulsion prepared by microchannel emulsication. Food Hydrocolloids 23, 600-610.

Klinkesorn U, McClements DJ. 2009. Influence of chitosan on stability and lipase digestibility of lecithin-stabilized tuna oil-in-water emulsions. Food Chem. 114, 1308-1315.

Knorr D. 1980. Effect of recovery methods on yield, quality and functional properties of potato protein concentrates. J. Food Sci., 45,1183-1186.

Krauss RM, Eckel RH, Howard B, Appel LJ, Daniels SR, Deckelbaum RJ. 2000. AHA dietary guidelines: revision 2000: a statement for healthcare professionals from the Nutrition Committee of the American Heart Association. Circulation 18, 2284-2299.

Laplante S, Turgeon L, Paquin P. 2005. Effect of pH, ionic strength, and composition on emulsion stabilizing properties of chitosan in a model system containing whey protein isolate. Food Hydrocolloids 19, 721729 .

McClements DJ. 2004. Role of hydrocolloids as emulsifiers in foods in Williams PA, Phillips, GO. (Eds.) Gums \& Stabilisers for the Food Industry 12, 381-393. Royal Society of Chemistry. Cambridge.

McClements DJ. 2005. Food emulsions. Principles, practices, and techniques (2nd ed.). Boca Raton: CRC Press.

McClements DJ, Weiss J. 2005. Lipid emulsion. In F. Shahidi (Ed.), Bailey's Industrial Oil and Fat Products (6th Ed.) John Wiley \& Sons, Inc.

Muzzarelli RAA. 2009. Chitin and chitosan hydrogels. In G. O. Philips, \& P. A. Williams (Eds.), Handbook of hydrocolloids (2nd ed.). (pp. 849-876) Boca Ratón: CRC Press.

Ogawa S, Decker EA, McClements DJ. 2003a. Influence of environmental conditions on stability of O/W emulsions containing droplets stabilized by lecithine chitosan membranes. J. Agr. Food Chem. 51, 55225527.

Ogawa S, Decker EA. McClements DJ. 2003b. Production and characterization of $\mathrm{O} / \mathrm{W}$ emulsions containing cationic droplets stabilized by lecithine chitosan membranes. J. Agr. Food Chem. 51, 2806-2812.

Ogawa S, Decker EA, McClements DJ. 2004. Production and characterization of $\mathrm{O} / \mathrm{W}$ emulsions containing droplets stabilized by lecithin-chitosane pectinmuti layered membranes. J. Agr. Food Chem. 52, 35953600.

Romero A, Beaumal V, David-Briand E, Cordobés F, Anton M, Guerrero A. 2011. Interfacial and O/W emulsions characterisation of potato protein isolate. J. Agr. Food Chem. 59, 9466-9474. 
Schmitt C, Sánchez C., Desobry-Banon S, Hardy J. 1998. Structure and techno functional properties of protein-polysaccharide complexes. Crit. Rev. Food Sci. 38, 689-753.

Speiciene V, Guilmineau F, Kulozik U, Leskauskaite D. 2007. The effect of chitosan on the properties of emulsions stabilized by whey proteins. Food Chem. 102, 1048-1054.

Tolstoguzov VB. 1991. In Gums and Stabilisers for the Food Industry 6, eds G.O. Philips, P.A. Williams \& D.J. Wedlock, Oxford University Press, Oxford, UK, 241-67.
Van Gelder WM, Vonk CR. 1980. Aminoacid composition of coagulable proteins and their nutritional quality. Potato Research 18, 469-478.

Van Koningsveld GA, Walstra P, Voragen AG, Kuijpers IJ, van Boekel MAJS Gruppen H. 2006. Effects of protein composition and enzymatic activity on formation and properties of potato protein stabilized emulsions. J. Agr. Food Chem. 54, 6419-642.

Recibido: $3 / 7 / 12$ Aceptado: 4/10/12 